\title{
Search performance without eye movements
}

\author{
RAYMOND KLEIN and MARK FARRELL \\ Dalhousie University, Halifax, Nova Scotia, Canada
}

\begin{abstract}
Visual search performance (with sets chosen to elicit both serial and parallel search patterns) under two conditions that precluded saccades was compared to the typical situation in which visual inspection of the array is possible. In one condition, the display duration was so brief that any saccades that were executed would be too late to bring the targeted portion of the array into the fovea. In the other, the display remained present until the subject's response, but eye position was monitored and trials with shifts in fixation were excluded from analysis. The latter condition produced search latencies that were nearly identical to those with free inspection. Brief exposure, in contrast, did not produce the pattern typical of serial search, presumably because of strategies induced to deal with the rapid decay of the visual array. It is concluded that saccadic eye movements play little role in the patterns of performance used to infer serial and parallel search, and that brief exposure is not a satisfactory technique for exploring the role of saccadic eye movements in visual search.
\end{abstract}

In recent years, we have seen great interest in two distinct patterns of performance in visual search. Under some conditions, search latencies appear to be relatively independent of the number of distractors. Under other conditions, search latencies increase linearly with the number of distractors in the display, and the slope of this function on target-present trials is $1 / 2$ that on target-absent trials. Many investigators refer to the former pattern as indicative of parallel search and to the latter pattern as indicative of serial self-terminating search, and we will therefore use the labels parallel and serial in order to facilitate discussion. To gain confidence in the validity of these labels, one must seek converging evidence (see, e.g., Klein, 1988). It should be noted that each of the patterns can be produced by both serial and parallel mechanisms (Townsend \& Ashby, 1983). Broadbent (1987) and others (e.g., Ward \& Mcdlelland, 1989), for example, have adapted to visual search a parallel activation model of the sort used by Ratcliff (1978) to explain the performance of subjects in a memory-search task (Sternberg, 1967).

In a variety of frameworks, including Treisman's feature-integration theory (Treisman, 1986; Treisman \& Gelade, 1980), the serial self-terminating search pattern is attributed to the sequential inspection of each item by an internal attentional system, a process that terminates when the target is found. ${ }^{1}$ In the typical search study, subjects are free to move their eyes, and-at least in serial search-they have time to make several saccades with the larger set sizes. This raises the possibility that it is not the number of covert shifts of an internal attentional "beam" that produces the linear reaction time (RT) increase in serial search, but rather the average number of

This research was supported by a Natural Sciences and Engineering Research Council of Canada grant to the first author. Requests for reprints should be addressed to Raymond Klein, Department of Psychology, Dalhousie University, Halifax, Nova Scotia B3H 4J1, Canada. saccades required to scan the display. In the present study, two manipulations were used to eliminate the possibility of scanning of the array with saccadic eye movements. If the serial pattern is correctly attributed to covert attentional shifts, then these manipulations should have little effect, and the typical patterns should be observed. On the other hand, if eliminating eye movements produces atypical patterns of search performance, then we may have to reexamine the assumed role of covert shifts of attention in producing serial search patterns.

\section{EXPERIMENT 1}

In Experiment 1, exposure of the array was limited to $180 \mathrm{msec}$, to prevent the subject from scanning the array. Since saccade initiation time is usually greater than $180 \mathrm{msec}$ (e.g., Klein, 1978), exposure duration is a frequently used device to eliminate the possibility that saccades will alter the position of a display on the retina (as in the split-field, laterality literature). It should be recognized, however, that brief exposures also decrease the quality of the information presented to the subject, and the consequent data limitations may have effects on perception and performance (e.g., Klein, 1982).

\section{Method}

Subjects. Eight university undergraduate volunteers participated for course credit.

Task and Stimuli. The subjects' task was to determine whether a target item was present in circular displays of 2,6 , or 10 items. The target and distractor items were circles, circles with gaps, or circles with lines. Target-distractor combinations were selected from Treisman (1986), to provide unambiguous examples of the performance patterns that have been used to infer parallel and serial selfterminating search (sample displays are shown in Figure 1). In the construction of the stimuli, gap position was determined randomly, but lines always transected the lower portions of the circles. In Treisman's (1986) report, search time for a circle with a gap or line in a background of whole circles is relatively unaffected by display size, so we will refer to these as parallel sets; search time for a 
whole circle in a background of circles with gaps or circles with lines increases linearly with display size, and the slope ratio for negative:positive trials is $1: 2$, so we will refer to these as serial sets.

The stimuli were displayed on a Tektronix 604 oscilloscope with P4 phosphor, in a dimly illuminated room. The viewing distance was $61.5 \mathrm{~cm}$, the radius of the circular items was approximately $.56^{\circ}$, and the radius of the imaginary circle on which they were randomly positioned (with equal spacing between all items in a display) was $2.4^{\circ}$. The gaps and lines subtended approximately $.5^{\circ}$. The exposure duration of the stimulus array was limited to $180 \mathrm{msec}$.

Procedure. The subjects were tested in a dimly illuminated room. The task was explained, and the subjects were instructed to press a switch with the right index or forefinger if they detected a target and to press a switch with the right middle finger if they did not detect a target. The subjects were then tested with each of the four stimulus sets in separate blocks. The target and distractor stimuli were described to the subject prior to each set, and the order of presentation of the four sets was varied between subjects. The subjects were tested on one practice block (36 trials) and one experimental block (96 trials), using each of the four display combinations (Figure 1). In the practice blocks, the display size was 6. The experimental blocks contained equal numbers of trials with display sizes of 2, 6, and 10. Within each display-size condition, half the trials contained targets and half did not. The subjects were allowed up to $3 \mathrm{sec}$ to respond.
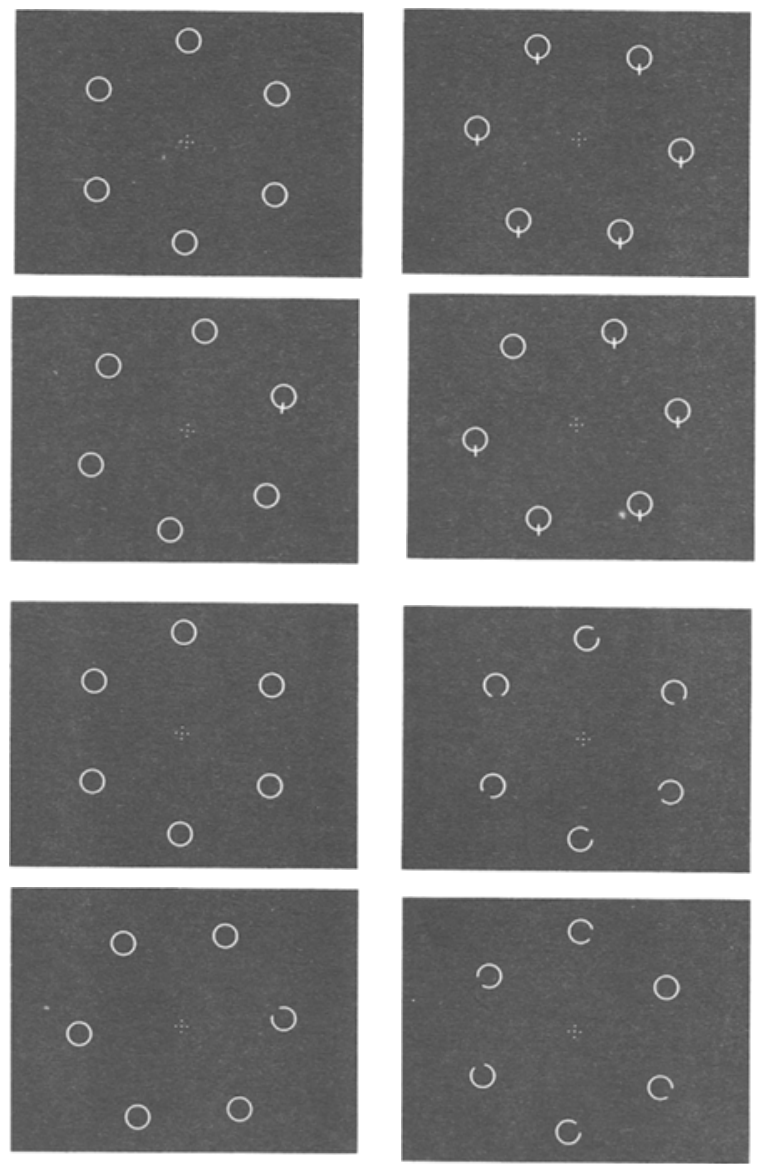

Figure 1. Examples of the stimulus displays for Set Size 6. Each quadrant represents examples of trials with (bottom) and without (top) targets from a particular set. The serial search sets are shown on the right, and the parallel search sets are on the left.

\section{Results}

Mean RT and error rates are shown in Figure $2 .{ }^{2}$ An analysis of variance (ANOVA) on the RT data revealed significant main effects of serial/parailel $[F(1,7)=31.88$, $p<.001]$, set size $[F(2,14)=33.31, p<.001]$, and whether a target was present or not (yes/no) $[F(1,7)=$ $8.58, p<.025]$. There was a significant two-way interaction between serial/parallel and set size $[F(2,14)=$ $9.51, p<.01]$. The three-way interaction between serial/parallel, set size, and yes/no was not significant $[F(2,14)=1.07]$. Inspection of the RT data reveals that performance with the serial search set does not conform to the typical pattern. Most striking are the nearly parallel curves for trials with and without targets in the serial search condition and the fact that the slopes of these curves are shallower than expected (about $26 \mathrm{msec} / \mathrm{item}$ ).

In the analysis of accuracy, the following main effects were significant: serial was less accurate than parallel $[F(1,7)=87.76, p<.001]$; accuracy decreased with set size $[F(2,14)=50.18, p<.001]$; there were more errors on trials with than on those without targets $[F(1,7)=$ $8.34, p<.025]$. The two-way and three-way interactions among these variables were also significant [serial/parallel $\times$ set size, $F(2,14)=77.72, p<.001$; serial/parallel $\times$ yes/no, $F(1,8)=8.41, p<.025$; set size $\times$ yes $/$ no, $F(2,14)=10.72, p<.005$; and serial/parallel $\times$ set size $\times$ yes/no, $F(2,14)=12.42, p<.001]$. Most of the variance underlying these effects and interactions is captured by the very dramatic increase in errors as set size increases in the serial condition with targets. There was also a significant interaction of stimulus type (line vs. gap), serial $/$ parallel, and yes/no $[F(1,7)=9.72, p<.025]$. It appears that there was a much stronger tendency for subjects to miss the circle targets (serial set) when the distractors were circles with gaps than when they were circles with lines, whereas stimulus type made little difference when circles were the distractors (parallel sets).

\section{Discussion}

The results of Experiment 1 suggest that when multiple fixations are precluded by the use of a short exposure, the pattern of search latencies with the serial sets is not typical. In particular, the search rates (slopes) on targetless and target trials are equivalent (rather than showing the typical 2:1 ratio), and they are dramatically less than when free inspection is permitted (see Experiment 2).

The rationale for the exposure-duration manipulation would seem to force the conclusion that multiple fixations may be at least partially responsible for the typical serial search pattern. However, a consideration of the error rates suggests an alternative explanation. Note that there is a dramatic increase in errors on target trials as set size increases from 2 to 10 . Following display offset, there is rapid loss of information about each of the potential targets in the display. Since subjects are searching for a target, when they do not find it (because it has decayed prior to inspection), they respond "no." The larger the set size, the more likely it is that the information will have decayed 


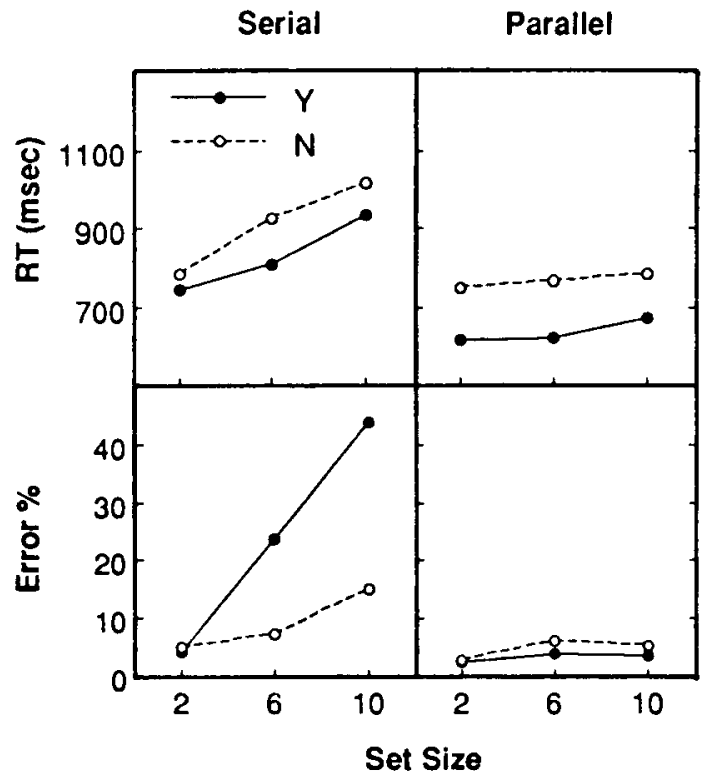

Figure 2. Mean RT and percent error as a function of array size for Experiment 1. Data from trials with targets $(Y)$ are shown in filled symbols, and data from trials without targets $(N)$ are shown in open symbols.

before the subject examines the critical location, thus producing the increase in "yes" errors (which are, of course, "no" responses). Moreover, because of the rapid decay, subjects should have an incentive to respond as quickly as possible. On targetless trials, this would reduce the slope, perhaps at the expense of accuracy with the larger set sizes.

Although this explanation is consistent with the observed pattern of results, it is by no means the only such explanation. For example, increases in set size might be accompanied by dramatic decreases in the criterion for initiating negative responses. It is not our goal in this paper to decide among these alternative descriptions. The main point we wish to draw from Experiment 1 is that the exposure-duration manipulation is flawed as a technique for evaluating the role of eye movements in producing the typical serial search pattern. As will be demonstrated in the remaining experiments, the performance patterns obtained in Experiment 1 are not due to the absence of eye movements per se, but rather to the special strategies adopted to deal with the short exposure.

\section{EXPERIMENT 2}

Although the notion that with brief displays rapid decay prevents subjects from examining all the locations and encourages speedy and less accurate responding is plausible, the possibility remains that multiple fixations do play a role in the serial search pattern. To permit a cleaner test of this proposition, in Experiment 2, two groups of subjects were tested with the display present for $3 \mathrm{sec}$ or until the subject responded, whichever came first. One condition (free) replicated the procedure used in typical visual search experiments: the subjects were free to move their eyes. In the other (fixed), the subjects were instructed to maintain fixation; eye position was monitored; and trials with eye movements were excluded. The latter condition permits us to eliminate multiple fixations without the undesirable effects produced by brief exposures.

\section{Method}

The methods for the two conditions of Experiment 2 were the same as those for Experiment 1, with the following exceptions: In both conditions, the array remained present for up to $3 \mathrm{sec}$ and was terminated by the subject's response. The fixation stimulus remained on for an additional 200-msec postresponse interval. In the fixed condition, horizontal and vertical eye position were monitored (Eye Trac 210) during the display of the array; the subjects were instructed to try to perform the search task while maintaining fixation; each block was preceded by a calibration procedure to ensure that shifts in gaze greater than about $.5^{\circ}$ vertically or horizontally would be easily detected; and trials with such gaze shifts were terminated, excluded from data analysis, and later repeated. The number of trials with eye movements during the display and after the response (during the subsequent 200 -msec interval) were noted for each block. Eight new volunteers served as subjects in each condition. The data from 1 subject in the eyes-fixed condition was not included in the following analyses, because his error rates were atypically high.

\section{Results}

Mean RT, error percentages, and standard deviations are shown in Figure 3. For each of these measures, the serial and parallel sets were subjected to separate ANOVAs, with fixed/free as a between-subjects variable, and type (gap/line), set size, and yes/no as within-subjects variables.

In the analysis of RT from the serial sets, only the effects of set size $[F(2,26)=96.27, p<.001]$, yes/no $[F(1,13)=68.47, p<.001]$, and their interaction $[F(2,26)=36.61, p<.001]$ were significant. It can be seen from inspection of Figure 3 that the expected pattern has been obtained: RT increases rapidly with set size, and the no:yes slope ratio is approximately $2: 1$. Precisely the same pattern is obtained both when the subjects are free to inspect the array and when their gaze is fixed $(F<1)$. The analysis of the parallel sets revealed significant main effects of type $[F(1,13)=11.67, p<.005]$, set size $[F(2,26)=25.06, p<.001]$, and yes/no $[F(1,13)=24.78, p<.001]$, as well as significant interactions between type and set size $[F(2,26)=12.26$, $p<.001]$, type and yes/no $[F(1,13)=14.7, p<.005]$, and set size and yes/no $[F(2,26)=13.92, p<.005]$. It can be seen in Figure 3 that the RT function is flat on target trials but increases significantly on targetless trials. This relationship was equally true in the fixed and free conditions $(F<1)$, but it did interact with whether the distractors were circles with gaps or lines $[F(2,26)=$ $16.09, p<.01]$. Inspection of the data for the gap and line stimulus sets separately (see Table 1) reveals that the increase in RT on targetless trials is due entirely to the gap stimuli, and that this pattern is responsible for all the effects involving stimulus type. The simplest interpreta- 


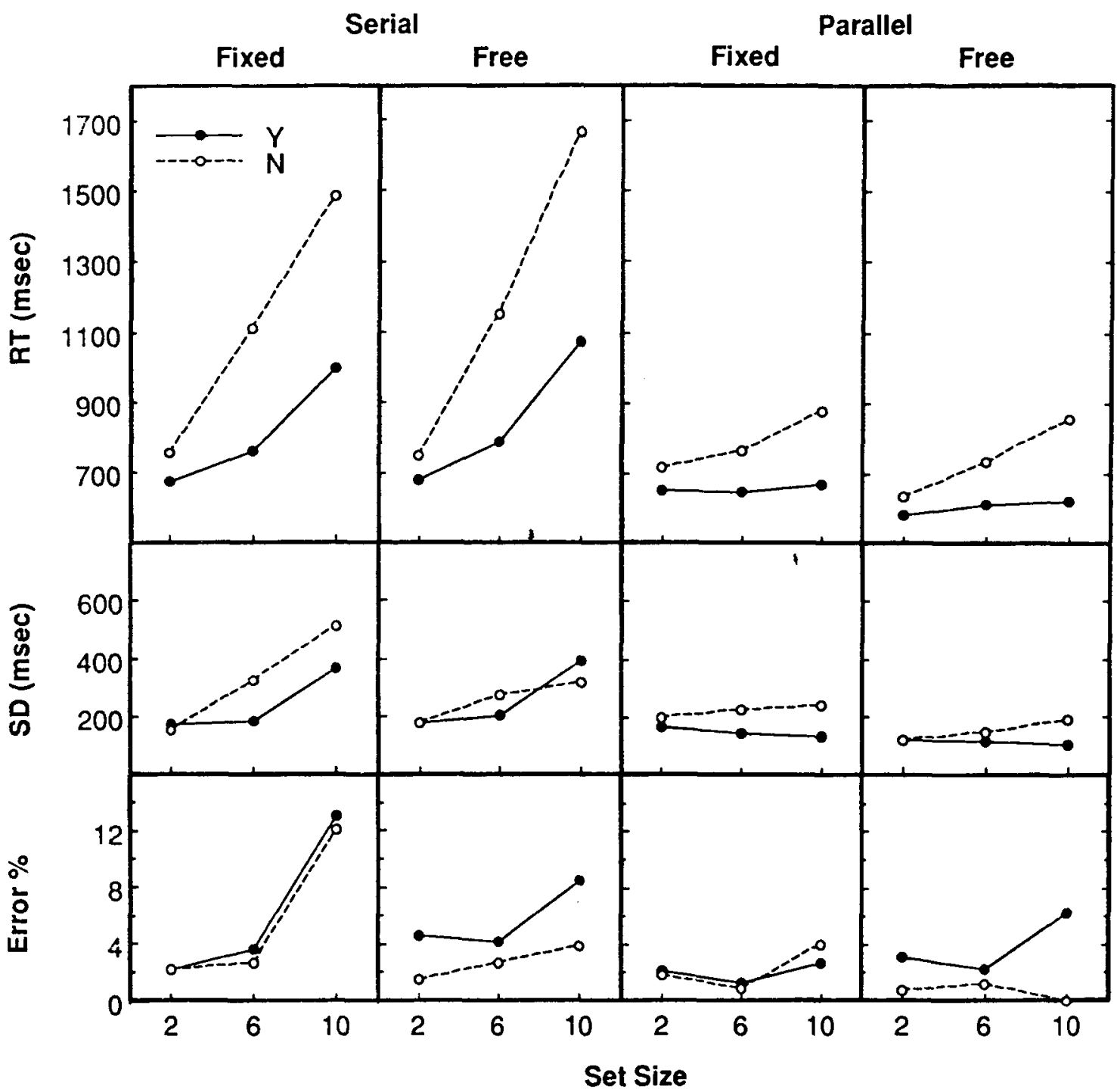

Figure 3. Mean RT, standard deviation of RT, and percent error as a function of array size for all conditions of Experiment 2. Data from trials with targets (Y) are shown in filled symbols, and data from trials without targets (N) are shown in open symbols.

tion of this finding is that the circle with a gap does pop out-hence the flat function when the target was present. However, given the low level of practice and switching between types of blocks, subjects hadn't learned this yet; when there is no target, there is a strong tendency to examine some distractors at least on some trials.

In the analysis of error percentages (i.e., accuracy) from the serial sets, there were significant main effects of type $[F(1,13)=8.36, p<.025]$ and set size $[F(2,26)=$ $35.75, p<.01]$, and there were also significant interactions between fixed/free and type $[F(1,13)=6.3, p<$ $.05]$ and between fixed/free and set size $[F(2,26)=10.41$, $p<.01]$. Inspection of the results in Figure 3 supports the following interpretation: When their eyes are fixed, subjects have difficulty with the largest set size. The ef- fects involving stimulus type are due to a higher error rate with the gap stimuli when the eyes were fixed (see Table 1). For the parallel sets there was a significant effect of set size $[F(2,26)=6.82, p<.01]$, and there were significant three-way interactions between fixed/free, type, and set size $[F(2,26)=3.85, p<.05]$ and between fixed/free, set size, and yes/no $[F(2,26)=3.54, p<$ $.05]$. The latter interactions appear to be due to relatively high error rates in particular cells (see Table 1), and they are not easy to explain.

In the analysis of standard deviations of RT from the serial sets, there were significant effects of set size $[F(2,26)=74.96, p<.01]$ and of yes/no $[F(1,13)=$ $23.39, p<.01]$, significant two-way interactions between fixed/free and yes/no $[F(1,13)=25.6, p<.01]$, 
Table 1

Performance Parameters (Mean Correct RT, Error Percentage, and Standard Deviation of Correct RT) for Each Stimulus Set and Condition of Experiment 2

\begin{tabular}{|c|c|c|c|c|c|c|c|c|c|c|c|c|c|}
\hline \multirow[b]{3}{*}{ Set Size } & \multirow[b]{3}{*}{ Response } & \multicolumn{12}{|c|}{ Stimulus Set } \\
\hline & & \multicolumn{3}{|c|}{ Serial/Gaps } & \multicolumn{3}{|c|}{ Parallel/Gaps } & \multicolumn{3}{|c|}{ Serial/Lines } & \multicolumn{3}{|c|}{ Parallel/Lines } \\
\hline & & RT & $\% \mathrm{E}$ & $S D$ & RT & $\% \mathrm{E}$ & $S D$ & RT & \#E & $S D$ & RT & $\% \mathrm{E}$ & $S D$ \\
\hline \multicolumn{14}{|c|}{ Fixed Condition } \\
\hline \multirow[t]{2}{*}{2} & Yes & 633 & 3.6 & 154 & 687 & 1.8 & 207 & 711 & .9 & 191 & 613 & 2.7 & 128 \\
\hline & No & 722 & 3.6 & 128 & 780 & 1.8 & 234 & 790 & .9 & 171 & 656 & 1.8 & 158 \\
\hline \multirow[t]{2}{*}{6} & Yes & 757 & 5.4 & 169 & 669 & 1.8 & 163 & 759 & 1.8 & 201 & 622 & .9 & 127 \\
\hline & No & 1,033 & 3.6 & 266 & 845 & 1.8 & 272 & 1,185 & 1.8 & 378 & 687 & .0 & 173 \\
\hline \multirow[t]{2}{*}{10} & Yes & 1,028 & 18.8 & 372 & 696 & 2.7 & 153 & 972 & 7.2 & 362 & 638 & 2.7 & 112 \\
\hline & No & 1,424 & 12.5 & 495 & 1,075 & 6.3 & 355 & 1,553 & 11.6 & 525 & 676 & 1.8 & 127 \\
\hline \multicolumn{14}{|c|}{ Free Condition } \\
\hline \multirow[t]{2}{*}{2} & Yes & 634 & 5.4 & 152 & 571 & 3.1 & 112 & 729 & 3.1 & 206 & 597 & 3.1 & 123 \\
\hline & No & 752 & .7 & 175 & 656 & .7 & 120 & 751 & 2.3 & 179 & 616 & .7 & 111 \\
\hline \multirow[t]{2}{*}{6} & Yes & 733 & 6.2 & 183 & 604 & 2.3 & 128 & 848 & 2.3 & 223 & 618 & 2.3 & 101 \\
\hline & No & 1,093 & 1.5 & 289 & 815 & .7 & 174 & 1,208 & 3.9 & 260 & 653 & 1.6 & 117 \\
\hline \multirow[t]{2}{*}{10} & Yes & 1,098 & 9.3 & 434 & 620 & 3.1 & 109 & 1,052 & 7.7 & 349 & 625 & 9.3 & 93 \\
\hline & No & 1,702 & 3.1 & 332 & 1,005 & .0 & 252 & 1,627 & 4.7 & 300 & 710 & .0 & 121 \\
\hline
\end{tabular}

Note-RT $=$ reaction time, $\% \mathrm{E}=$ percent error, $S D=$ standard deviation. $\mathrm{RTs}$ are given in milliseconds.

fixed/free and set size $[F(2,26)=3.82, p<.05]$, and set size and yes/no $[F(2,26)=5.94, p<.01]$, and significant three-way interactions among fixed/free, type, and yes/no $[F(1,13)=5.68, p<.05]$, and among fixed/free, set size, and yes/no $[F(2,26)=6.25, p<.01]$. It can be seen from inspection of Figure 3 that the latter interaction is due to the fact that with the eyes free, standard deviations increased faster on yes than on no trials, whereas with the eyes fixed, the reverse was true. It should be noted, however, that there are no differences between fixed/free on yes trials; the interaction is due completely to the large increase in variability on no trials when the eyes are fixed.

For the parallel sets, there were significant main effects of type (gap vs. line) $[F(1,13)=11.95, p<.01]$ and of yes/no $[F(1,13)=10.21, p<.01]$, and there were significant interactions between type and set size $[F(2,26)=4.66, p<.025]$, set size and yes/no $[F(2,26)$ $=4.89, p<.025]$, and type, set size, and yes/no $[F(2,26)=6.96, p<.001]$. The major source of variance underlying these effects derives from the increase in variance with set size when there is no target with the gap set (see Table 1). In the remaining conditions, standard deviations are relatively consistent. This pattern is quite consistent with the explanation for the RT interaction involving type described above.

The subjects averaged about 18 trials with eye movements ( 15 during the stimulus and 3 during the 200 -msec postresponse interval) during each block (recall that these trials were replaced, to provide a total of 96 usable trials per block). An ANOVA was performed to determine whether the frequency of eye movements was affected by stimulus type (line/gap) or stimulus set (serial/parallel). No effects were significant (all $F \mathrm{~s}<1$ ).

\section{Discussion}

When multiple fixations are precluded by monitoring eye position, the pattern of search latencies with the serial search sets appears to be typical, and it does not differ from that obtained when free inspection is permitted. The no:yes slope ratio is approximately $2: 1$ (87 msec/item vs. $43 \mathrm{msec} / \mathrm{item}$ ), and the implied search rate is in the range reported by Treisman and others. It is clear both from comparison of the serial data in Figures 2 and 3 and from the subsequent analyses that restricting visual inspection by means of a brief exposure produces a substantially different pattern of search performance than is obtained when free inspection is permitted. In contrast, when exposure duration is not manipulated and shifts in gaze are prevented by eye-position monitoring, the RT pattern is indistinguishable from that with free inspection.

Although the RT pattern with the serial sets is identical whether the eyes were free or fixed, there are two differences between these conditions, both in the same cell, that merit some attention. First, with Set Size 10 and no target, errors were much higher when eye movements were precluded (12.1 vs. 3.9). Second, variability in this cell was much higher with the eyes fixed than with them free-so much so, that with the eyes fixed, standard deviations did not show the yes/no crossover pattern. These differences might be taken as evidence that eye movements do play a role in producing data indicative of the stan. dard serial search strategy. We do not believe that this view is supported by the overall pattern of our findings, however. Note that when the eyes are free to move, variability for trials with targets increases faster than for trials without targets. The greater increase in variability on target trials is, according to the serial, self-terminating model, due to the inclusion of a greater range of finish- 
ing times as set size increases (see Townsend \& Ashby, 1983; Proposition 77). In comparison with eyes fixed, it is variability on trials without targets that differs; the variability on target trials was exactly the same in the two conditions. Consideration of the accuracy difference between the two experiments provides an explanation consistent with the serial, self-terminating model for both conditions. Note that when the eyes were fixed, there was a large increase in errors (relative to eyes free) in the target-absent, Set Size 10 condition. This condition also showed the variance difference. We believe that two factors are together responsible for both the higher errors and the increased variance in this condition. First, the subjects found it difficult to maintain fixation for long periods of time in the eyes-fixed condition, and when a shift in gaze seemed imminent, they terminated the trial-andsearch process by guessing. Such a strategy would have been encouraged by the fact that trials with eye movements were later repeated, thus prolonging the experiment. This strategy is likely to have affected the cells with the slowest times the most. The effect would have been threefold, consisting of an increase in errors, an increase in variance, and a decrease in mean RT. Also adding to inaccuracy and variance in this condition could have been the poorer quality of information available about the potential targets when the 10 parafoveal items could not be fixated. This would also have tended to counteract the trend to reduced RT just mentioned. The ad hoc nature of this explanation should not detract from the conclusion that is dictated by the nearly identical latency patterns: The RT patterns typically used to infer serial, selfterminating search cannot be attributed to scanning of the stimulus array by saccadic eye movements.

As in the serial condition, whether eye position was fixed or free also had no effect on the RT pattern with the parallel sets. Nevertheless, the data from the parallel gap sets were somewhat anomalous. When the target was a circle with a gap (parallel set), yes RT did not vary with set size, but there was a fairly steep increase in RT on targetless trials ( $30 \mathrm{msec} / \mathrm{item}$ and $45 \mathrm{msec} / \mathrm{item}$, in the two conditions of the experiment). The simplest interpretation of this finding is that the circle with a gap does pop out, but because of the low level of practice with these particular stimuli, subjects do not realize this yet. On trials with targets, RT is thus unaffected by set size, but on targetless trials, subjects may frequently engage in serial search. In contrast, the pop-out experience with the line stimuli may be much more salient, permitting subjects to use the failure of pop-out to signal a "no" response.

\section{CONCLUSIONS}

For the purpose of exploring the role of normally occurring eye movements, exposure duration is not a particularly good way to control fixation, because subjects adopt special strategies to deal with the rapidly decaying icon.
It should be noted that Treisman and Gormican (1988, Figure 14) also attempted to use brief exposures to assess the potential role of multiple fixations in producing serial search patterns. They used a restricted range of set sizes (1-6 items), because they anticipated that error rates would be too high with the larger sizes. (Indeed, even with this restricted range, 6 out of 14 subjects could not keep errors below $33 \%$ in all conditions). Their RT pattern was fairly typical of serial search (with a 1.75:1 absent:present slope ratio), so they concluded that multiple fixations do not play a causal role in this pattern. ${ }^{3}$ On the basis of Experiment 2, we agree with this conclusion, but we would not be confident in drawing it from our own exposure-duration manipulation. Nevertheless, comparison of our results with those of Treisman and Gormican reveals a remarkably similar pattern. If we look only at the results from our Set Sizes 2 and 6, our slope ratio is approximately $2: 1$, and with Set Size 6, our miss rate is, like theirs, unusually high (about $24 \%$ ). Only our data from Set Size 10 make our pattern different from theirs, and they purposely excluded set sizes above 6 .

The pattern of search latency with the serial and parallel sets is almost unaffected when scanning eye movements are eliminated by the monitoring of eye position together with the provision of feedback on gaze shifts. Although the accuracy and standard deviations are affected in the eyes-fixed condition (Set Size 10, no target), the particular pattern obtained is explained most easily by assuming that subjects prematurely terminate search, and then guess, on a proportion of trials (perhaps because they sense a trial-terminating eye shift or blink). Whether or not this explanation is correct, we can conclude that saccadic eye movements play little role in producing the RT patterns. Internal mechanisms, possibly covert shifts of attention, are responsible for the linear increase in RT with set size and the 2:1 no:yes slope ratio obtained with the serial search sets. Alternatively, the patterns of search performance we have obtained might be accommodated within a parallel activation model of the sort proposed by Ratcliff (1978) for memory search and extended by Broadbent (1987) and Ward and McClelland (1989) to visual search. In the context of such models, variables such as the choice of different target-distractor pairings, the exposure duration, and whether subjects have the opportunity to inspect the stimulus objects with multiple fixations would be associated with parameter selection in such a way that the patterns of performance (RT, accuracy, and standard deviations) we and others have observed can be closely simulated.

\section{REFERENCES}

Broadbent, D. E. (1987). Simple models for experimentable situations. In P. E. Morris (Ed.), Modelling cognition. London: Wiley. KLEIN, R. M. (1978). Chronometric analysis of saccadic eye movements: Reflexive and cognitive control. In D. Landers \& R. Christina (Eds.), The psychology of motor behavior and sport: 1977. Champaign, IL: Human Kinetics Publishers. 
KLEIN, R. M. (1982). Patterns of perceived similarity cannot be generalized from long to short exposure durations and vice versa. Perception \& Psychophysics, 32, 15-18.

KLEIN, R. M. (1988). Inhibitory tagging system facilitates visual search. Nature, 334, 430-431.

Pashler, H. (1987). Detecting conjunctions of color and form: Reassessing the serial search hypothesis. Perception \& Psychophysics, 41, 191-201.

RAtCliff, R. (1978). A theory of memory retrieval. Psychological Review, 85, 59-108.

STERNBERG, S. (1967). Two operations in character recognition: Some evidence from reaction-time measurements. Perception \& Psychophysics, 2, 45-53.

ToWNSEnd, J. T., \& AshBy, F. G. (1983). Stochastic modelling of elementary psychological processes. New York: Cambridge University Press.

Treisman, A. (1986). Features and objects in perception. Scientific American, 225, No. 11, 114-125.

Treisman, A., Gelade, G. (1980). A feature-integration theory of attention. Cognitive Psychology, 12, 97-136.

Treisman, A. Gormican, S. (1988). Feature analysis in early vision: Evidence from search asymmetries. Psychological Review, 95 , $15-48$.

WARD, R., \& MCClelland, J. L. (1989). Conjunctive search for one and two identical targets. Journal of Experimental Psychology: Human Perception \& Performance, 15, 664-672.

\section{NOTES}

1. Pashler (1987) has described a hybrid model in which groups of items are searched in sequence, with parallel search over the items in a group. In our Experiment 2, there was no evidence for the pattern of results that Pashler relies on to support this proposal (that is, we did not obtain parallel yes and no RT functions with set sizes restricted to 2 and 6). Since the boundary conditions for obtaining Pashler's pattern are not known, it would be premature for us to address his model in this paper.

2. The data files from this experiment were lost before within-subjects standard deviations could be calculated for each condition. Although in principle this information is desirable, given the very high error rates with the largest set sizes, we doubt that the standard deviations would have been reliable.

3. They did find, however, that the search rates were significantly faster than when free inspection was permitted. However, the increasing miss rate with increases in set size suggested that subjects did not search all the items with the larger displays. Treisman and Gormican therefore used the miss rates with the larger set sizes to compute the effective number of items "searched," and, given these adjusted set sizes, the search rates with brief displays were the same as with free inspection.

(Manuscript received October 17, 1988; revision accepted for publicaton May 22, 1989.) 